(2) Open Access Full Text Article

\title{
Upper eyelid retraction disclosed after edrophonium chloride administration in a patient with Graves' orbitopathy and myasthenia gravis
}

This article was published in the following Dove Press journal:

Clinical Ophthalmology

26 May 2012

Number of times this article has been viewed

Hyera Kang ${ }^{1,2}$

Yasuhiro Takahashi'

Masayoshi Iwaki'

Shinichi Asamura ${ }^{3}$

Hirohiko Kakizaki'

'Department of Ophthalmology, Aichi Medical University, Nagakute, Aichi, Japan; ${ }^{2}$ Department of Ophthalmology, Presbyterian Medical Center, Jeonju, Korea; ${ }^{3}$ Department of Plastic and Reconstructive Surgery, Kinki University School of Medicine, Osaka-Sayama, Osaka, Japan
Correspondence: Hirohiko Kakizaki Department of Ophthalmology, Aichi Medical University, Nagakute, Aichi 480-II95, Japan

Tel $+8|56| 6233 \mid I$ ext $218 \mid$

Fax +8I 56I 637255

Email cosme@dI.dion.ne.jp
Abstract: Patients with Graves' orbitopathy have a higher probability of myasthenia gravis than does the normal population. Overlapping clinical features cause diagnostic confusion in such a situation. We herein report a patient with Graves' orbitopathy and myasthenia gravis (GO-MG) with normal left eyelid height, but in whom upper eyelid retraction was shown after edrophonium chloride administration. Upper eyelid retraction in GO-MG is occasionally masked by a myasthenia effect. The upper eyelid height must be carefully monitored in patients with Graves' orbitopathy to detect the presence of concomitant myasthenia gravis.

Keywords: Graves' orbitopathy, myasthenia gravis, eyelid retraction, edrophonium chloride

\section{Introduction}

Patients with Graves' orbitopathy have a higher probability of myasthenia gravis than the normal population. ${ }^{1}$ According to a large retrospective case series, myasthenia gravis was diagnosed in two of 150 patients with Graves' orbitopathy $(1.3 \%){ }^{2}$ Patients with Graves' orbitopathy commonly show eyelid retraction and/or hypotropia and/or esotropia, as well as exophthalmos, lagophthalmos, exposure keratopathy, and less frequently, compressive optic neuropathy. ${ }^{3}$ However, patients with myasthenia gravis often demonstrate ptosis and/or exotropia, but a consistent pattern of eye movement disturbances is usually not present. ${ }^{3}$ Upper eyelid retraction in Graves' orbitopathy and myasthenia gravis (GO-MG) is frequently camouflaged, and these patients may show a normal upper eyelid height. We herein report a patient with GO-MG with left normal eyelid height, but in whom the upper eyelid retraction was disclosed after edrophonium chloride administration.

\section{Case report}

A 46-year-old man with Graves' disease, well controlled by thiamazole $5 \mathrm{mg} /$ day, was referred to our clinic to treat his Graves' orbitopathy. Although both eyes had exophthalmos (20 mm OD, $19 \mathrm{~mm}$ OS; normal range, $<17 \mathrm{~mm})^{4}$, he demonstrated a normal left eyelid height and right ptosis, ie, margin reflex distance-1, $3.5 \mathrm{~mm}$ OS and $0.5 \mathrm{~mm}$ OD, respectively (Figure 1). His primary eye position was exotropic, dominantly on his right side, and the upward gaze of the right eye was severely restricted (Figure 2). Coronal computed tomography of the orbits showed bilateral enlargement of the inferior rectus muscle, medial rectus muscle, superior oblique muscle, and superior rectus muscle-levator muscle complex (Figure 3A). Axial computed tomography demonstrated bilateral medial and lateral recti muscle enlargement (Figure 3B). Localization of these 


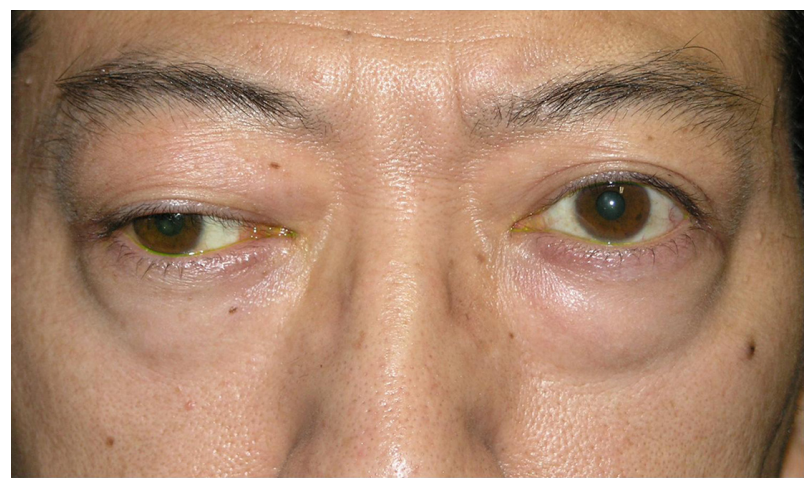

Figure I Patient photograph before edrophonium chloride administration.

muscle involvements did not match the characteristics of the eye movement disorder (Figure 3C).

Because the aforementioned findings raised suspicion for the presence of both Graves' orbitopathy and myasthenia gravis, we performed an edrophonium chloride test. ${ }^{5}$ The margin reflex distance-1 had improved to $2.5 \mathrm{~mm}$ OD and $8.0 \mathrm{~mm}$ OS (Figure 4), although the eye movement had not clearly improved.

Some days later, the result of a serum acetylcholine receptor antibody assay was positive $(1.8 \mathrm{nmol} / \mathrm{L}$; normal, $<0.2 \mathrm{nmol} / \mathrm{L}$ ). Because the patient could completely close his left eye, we prescribed pyridostigmine bromide $180 \mathrm{mg} /$ day, ${ }^{5}$ and the eyelid height stabilized. The exophthalmos did not change after the administration of pyridostigmine bromide.

\section{Comment}

The association between Graves' orbitopathy and myasthenia gravis has long been recognized. ${ }^{2,6}$ Although it is not difficult to differentiate clinically between simple Graves' orbitopathy and myasthenia gravis, overlapping clinical features, such
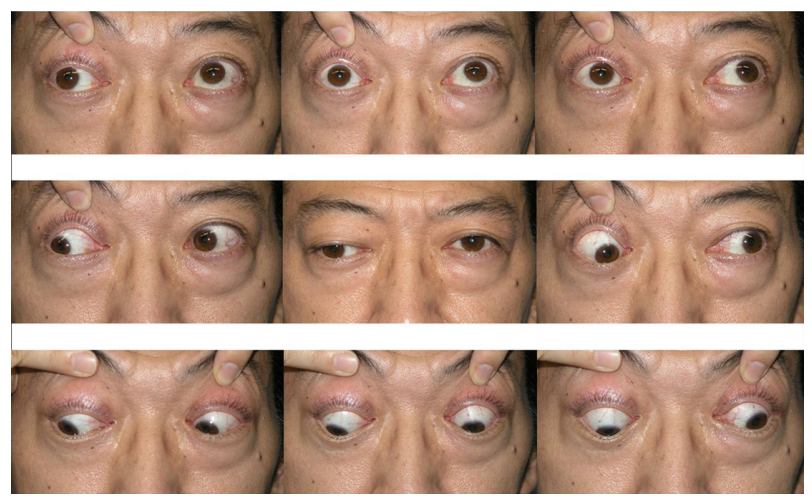

Figure 2 Patient's eye positions in nine directions of gaze before edrophonium chloride administration.

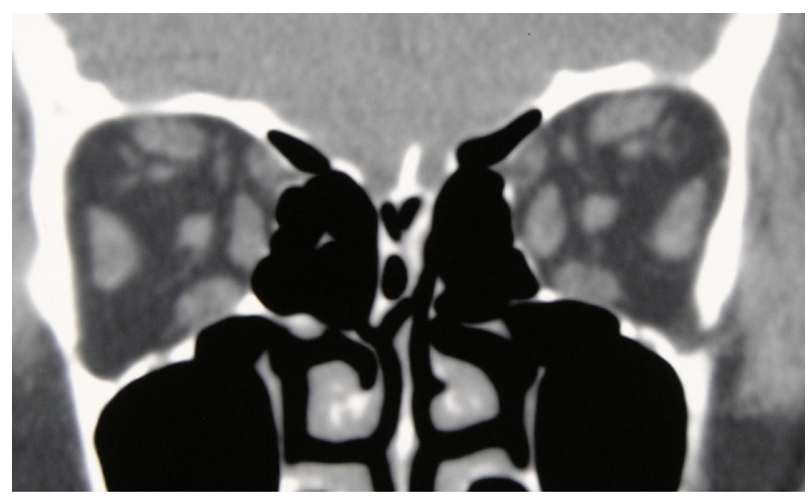

Figure 3A Coronal computed tomography of the orbits shows bilateral enlargement of the inferior rectus muscle, medial rectus muscle, superior oblique muscle, and superior rectus muscle-levator muscle complex.

as upper eyelid height and/or eye movement disturbances occasionally cause diagnostic confusion, especially in patients with normal thyroid function.

The most frequent sign of Graves' orbitopathy is upper eyelid retraction, ${ }^{7}$ which is caused by overaction of the Müller's muscle because of excess catecholamines and cicatricial contraction of the levator muscles after the inflammatory process. ${ }^{7}$ For the overaction, topical guanethidine therapy is sometimes effective. ${ }^{8}$ For the contraction, steroid administration and botulinum toxin injection are indicated during the active phase, and surgical correction is necessary in the static phase. ${ }^{7}$

Graves' orbitopathy also shows eye movement disturbances, which have a restrictive myopathy pattern. ${ }^{3}$ The inferior and medial rectus muscles are frequently involved, and imaging discloses their hypertrophy when in the active phase.

On the other hand, ptosis and diplopia are present in about $90 \%$ of patients with myasthenia gravis, and these ocular

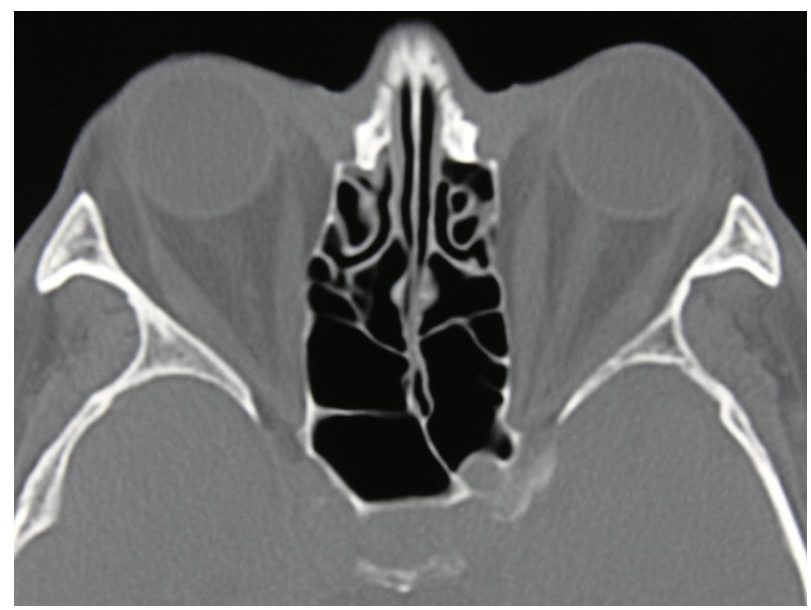

Figure 3B Axial computed tomography demonstrates bilateral medial and lateral recti muscle enlargement. 


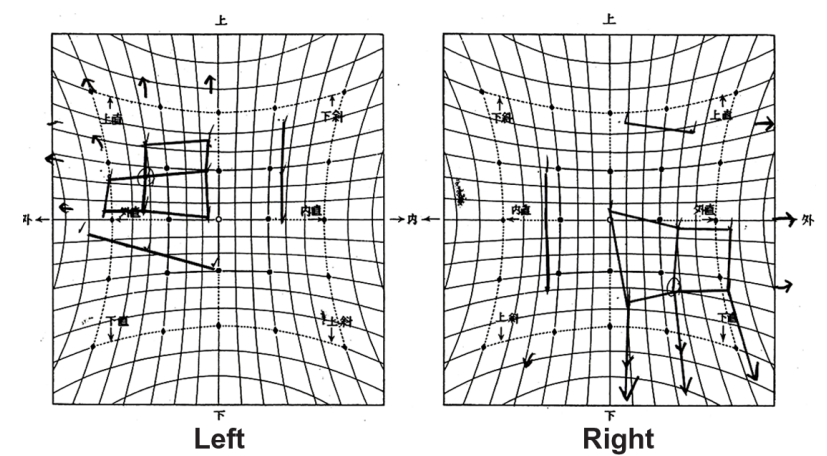

Figure $3 \mathrm{C}$ Hess screen chart before edrophonium chloride administration.

symptoms are an initial complaint in $20 \%$ of patients with myasthenia gravis. ${ }^{9-11}$ Muscle hypertrophy does not exist on imaging in myasthenia gravis. ${ }^{12}$ No particular predilection of extraocular muscle involvement is shown in myasthenia gravis. However, solitary paresis usually occurs, in the form of an adduction deficit resulting in exotropia, which was the case in the present patient. Exotropia is more common in ocular myasthenia gravis. ${ }^{3}$

In spite of the obvious differences between Graves' orbitopathy and myasthenia gravis, each condition shares several features, potentially making it difficult to reach a correct diagnosis. Upper eyelid ptosis, typically suggestive of myasthenia gravis, may be seen with Graves' orbitopathy as a result of levator myopathy, ${ }^{13}$ apical compression, ${ }^{14,15}$ and pseudoptosis secondary to upper eyelid retraction in the fellow eye. ${ }^{16}$ However, if ptosis develops in a patient with Graves' orbitopathy, simultaneous myasthenia gravis should be considered, ${ }^{17}$ although ocular myasthenia gravis with orbital pain may imply orbital inflammation. ${ }^{18}$

In our patient, the right ptosis and exotropia suggested concomitant myasthenia gravis, which motivated us to perform an edrophonium chloride test. The left upper eyelid, appearing to have a normal height, was retracted

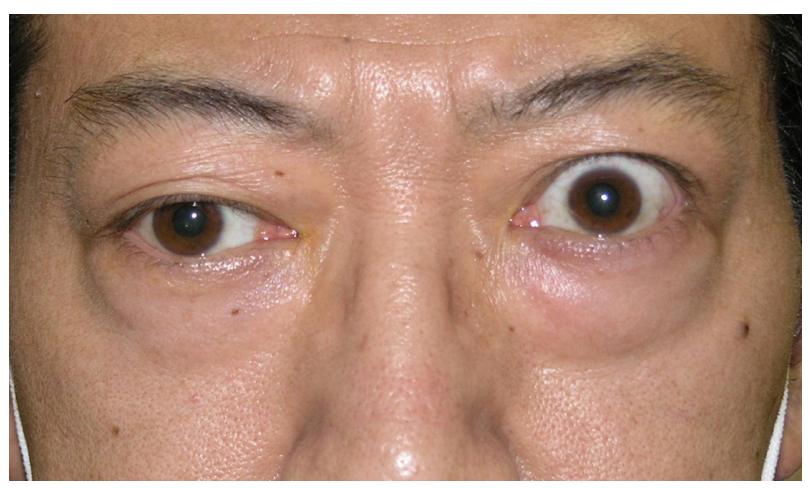

Figure 4 After edrophonium chloride administration. to margin reflex distance-1 $8.0 \mathrm{~mm}$, and the right side, regarded as ptosis, moved to margin reflex distance- $12.5 \mathrm{~mm}$ (Figure 4). This examination demonstrated that the left upper eyelid originally had an upper eyelid retraction that was camouflaged by the ocular myasthenia gravis.

Treatment of our patient was complicated because of laterality and strabismus induced by myasthenia gravis. When the eyelid height stabilizes after medication with improvement of strabismus, a sling surgery may be performed on the right upper eyelid. However, if strabismus persists, this strategy must be abandoned. When left upper eyelid retraction is conspicuous with lagophthalmos, left upper eyelid lengthening surgery may be performed. ${ }^{19}$

In conclusion, upper eyelid retraction in patients with GO-MG may be camouflaged by a myasthenia effect. The upper eyelid height must be carefully monitored in patients with Graves' orbitopathy to detect the presence of concomitant myasthenia gravis.

\section{Disclosure}

The authors report no conflicts of interest in this work.

\section{References}

1. Victor M, Ropper AH. Adams and Victor's Principles of Neurology, 8th ed. New York, NY: McGraw-Hill; 2005.

2. Cruz AAV, Akaishi PMS, Vargas MA, de Paula SA. Association between thyroid autoimmune dysfunction and non-thyroid autoimmune diseases. Ophthal Plast Reconstr Surg. 2007;23(2):104-108.

3. Vargas ME, Warren FA, Kupersmith MJ. Exotropia as a sign of myasthenia gravis in dysthyroid ophthalmopathy. Br J Ophthalmol. 1993;77(12):822-823.

4. Preechawai P. Anthropometry of eyelid and orbit in four southern Thailand ethnic groups. J Med Assoc Thai. 2011;94(2):193-199.

5. Vincent A, Palace J, Hilton-Jones D. Myasthenia gravis. Lancet. 2001;357(9274):2122-2128.

6. Marino M, Barbesino G, Manetti L, et al. Mild clinical expression of myasthenia gravis associated with autoimmune thyroid diseases. J Clin Endocrinol Metab. 1997;82(2):3905-3906.

7. Dutton JJ. Surgical management of eyelid retraction in thyroid eye disease. In: Dutton JJ, Haik BG, editors. Thyroid Eye Disease: Diagnosis and Treatment. New York, NY: Marcel Dekker Inc; 2002.

8. Gay AJ, Wolkstein MA. Topical guanethidine therapy for endocrine lid retraction. Arch Ophthalmol. 1966;76(3):364-367.

9. Cleary PE. Ocular manifestations of myasthenia gravis. Br Orthopt $J$. 1973;30:38-51.

10. Acers TE. Ocular myasthenia gravis mimicking pseudointernuclear ophthalmoplegia and variable esotropia. Am J Ophthalmol. 1979; 88(3 Pt 1):319-321.

11. Gunji K, Skolnick C, Bednarczuk T, et al. Eye muscle antibodies in patients with ocular myasthenia gravis: possible mechanism for eye muscle inflammation in acetylcholine-receptor antibody-negative patients. Clin Immunol Immunopathol. 1998;87(3):276-281.

12. Friedman DI. Myasthenia gravis. In: Yanoff M, Duker JS, editors. Ophthalmology, 2nd ed. St Louis, MO: Mosby; 2004.

13. Burde RM. Graves' ophthalmopathy and the special problem of concomitant ocular myasthenia gravis. Am Orthopt. 1990;40:37-50.

14. Naseem M, Donker DL, Paridaens D. Blepharoptosis as a sign of severe Graves' orbitopathy. Eye (Lond). 2009;23(8):1743-1744. 
15. Weis E, Heran MKS, Jhamb A, et al. Clinical and soft-tissue computed tomographic predictors of dysthyroid optic neuropathy: refinement of the constellation of findings at presentation. Arch Ophthalmol. 2011;129(10):1332-1336.

16. Batocchi AP, Evoli A, Majolini L, et al. Ocular palsies in the absence of other neurological or ocular symptoms analysis of 105 cases. J Neurol. 1997;244(10):639-645.

17. Yaman A, Yaman H. Ocular myasthenia gravis coincident with thyroid ophthalmopathy. Neurol India. 2003;51(1):100-101.
18. Kusuhara T, Nakajima M, Imamura A. Ocular myasthenia gravis associated with euthyroid ophthalmopathy. Muscle Nerve. 2003;28(6):764-766.

19. Kakizaki H, Zako M, Iwaki M. Lower eyelid lengthening surgery targeting the posterior layer of the lower eyelid retractors via a transcutaneous approach. Clin Ophthalmol. 2007;1(2):141-147.

\section{Publish your work in this journal}

Clinical Ophthalmology is an international, peer-reviewed journal covering all subspecialties within ophthalmology. Key topics include: Optometry; Visual science; Pharmacology and drug therapy in eye diseases; Basic Sciences; Primary and Secondary eye care; Patient Safety and Quality of Care Improvements. This journal is indexed on

\section{Dovepress}

PubMed Central and CAS, and is the official journal of The Society of Clinical Ophthalmology (SCO). The manuscript management system is completely online and includes a very quick and fair peer-review system, which is all easy to use. Visit http://www.dovepress.com/ testimonials.php to read real quotes from published authors. 